\title{
L'Association médicale mondiale a révisé la Déclaration de Genève
}

\author{
Urban Wiesing ${ }^{a}$, Ramin Parsa-Parsib \\ a Prof. Dr méd. Dr phil., Directeur de I'Institut d'éthique et d'histoire de la médecine de l'Université de Tübingen; ${ }^{b}$ Dr méd., MPH, Chef du Département des \\ affaires étrangères de la Chambre médicale fédérale allemande, Berlin; membre du Comité de l'Association médicale mondiale, chef du Groupe de travail \\ pour la révision de la Déclaration de Genève
}

Nous sommes reconnaissants à l'Association médicale mondiale d'avoir permis la traduction de ce texte important
A l'occasion de sa $68^{\mathrm{e}}$ Assemblée générale, qui s'est tenue le 14 octobre 2017 à Chicago, l'Association médicale mondiale a adopté une version révisée de la Déclaration de Genève qui apporte quelques modifications et ajouts importants. ${ }^{1}$ L'un des documents les plus importants de l'Association médicale mondiale a ainsi été adapté, un document dont l'importance peut être comparée à celle de la Déclaration d'Helsinki sur les principes éthiques pour la recherche médicale sur des êtres humains.

La Déclaration de Genève a une longue histoire: elle a été adoptée en 1948, à l'occasion de la $2^{\mathrm{e}}$ Assemblée générale de l'Association médicale mondiale, suite à la Seconde Guerre mondiale et aux atrocités réalisées du fait de la participation des médecins au régime nazi. Ce serment a été élaboré afin de permettre le rétablissement de la confiance des patients à l'égard de la profession médicale. Il a légèrement été révisé en 1968 (Sydney), 1983 (Venise) et 1994 (Stockholm) et certaines de ses parties ont fait l'objet d'un toilettage linguistique en 2005 et 2006. Il résume les principes éthiques fondamentaux de l'activité médicale. Son titre français, Serment d'Hippocrate, formule de Genève, fait référence à la longue tradition dans laquelle s'inscrit le serment.

\section{Principes de la révision}

L'Association médicale mondiale a nommé les associations médicales d'Inde, de Turquie, d'Israël, de Suède et des Etats-Unis comme membres du groupe de travail responsable de la révision et en a attribué la gestion à l'association médicale allemande. Cela est particulièrement important compte tenu de l'évolution historique de la Déclaration de Genève. La composition du groupe de travail comprenait délibérément des membres ayant des contextes culturels, religieux et ethniques différents. Un délai de traitement de deux ans avait été fixé du fait de l'importance capitale du serment pour l'Association médicale mondiale.

Le groupe de travail a rapidement convenu que la révision de la Déclaration de Genève ne devait concerner ni son caractère, ni sa portée, ni son orientation ou ses destinataires. Seuls des changements présentant des arguments solides devaient par ailleurs être réalisés. Les experts intéressés et les parties prenantes ont eu l'occasion de participer à la révision dans le cadre d'une consultation publique, en proposant des amendements. Les membres de l'Association médicale mondiale ont en outre eu plusieurs occasions de commenter le projet amendé. Le groupe de travail a examiné toutes les observations en fonction des principes susmentionnés.

\section{Ajouts et amendements}

La révision a apporté des ajouts et changements significatifs à divers égards. La phrase selon laquelle les médecins doivent respecter l'autonomie de leurs patients est très certainement l'ajout le plus important. Tout comme le Serment d'Hippocrate, la Déclaration de Genève n'avait jusqu'ici jamais fait état du respect de l'autodétermination du patient. Cela était cependant en contradiction avec de nombreux autres documents de l'Association médicale mondiale, tels que la Déclaration d'Helsinki, qui appelle clairement et de façon précise la recherche clinique à respecter l'autonomie des patients. Le serment éthique principal de la profession médicale (dont l'original est en anglais) a, avec cet amendement, désormais repris l'autodétermination des patients: «I WILL RESPECT the autonomy and dignity of my patient.»

La révision a également tenté d'actualiser certaines formulations. Le terme «consecrate» (consacrer), ayant, en anglais, une certaine connotation religieuse, a ainsi été remplacé par le terme "dedicate» (dédier): «I SOLEMNLY PLEDGE to ensecrate dedicate my life to the service of humanity.» Le lien avec la tradition de la profession a également été déterminé de façon plus sobre: "I WILL FOSTER MAINTAIN by all means in my power, the honour and noble traditions of the medical profession.» L'expression "MY COLLEAGUES will be my sisters and brothers» a également été perçue comme étant obsolète et a par conséquent été supprimée et remplacée dans la phrase par «colleagues and students»: «I WILL 
GIVE to my teachers, colleagues, and students the respect and gratitude that is their due.»

L'extension aux étudiants résulte notamment d'une intervention de la Fédération internationale des associations d'étudiants en médecine (International Federation of Medical Students' Associations, IFMSA). Ses représentants ont fait savoir à l'Association médicale mondiale que les étudiants devaient faire preuve de respect à l'égard de leurs professeurs, mais que le contraire ne ressortait pas de la version en vigueur. La IFMSA a ainsi, à juste titre, demandé la réciprocité, qui était déjà incluse dans le Serment d'Hippocrate âgé de plus de 2500 ans. Ceci est maintenant exprimé par la nouvelle formulation.

La révision adoptée adresse le traitement du savoir. La Déclaration de Genève impose dorénavant au médecin de partager son savoir afin de servir les patients et les soins de santé: «I WILL SHARE my medical knowledge for the benefit of the patient and the advancement of healthcare.»

Nouvelle est également une phrase se référant au médecin, à sa santé et à son bien-être. De nombreuses études ont mis en évidence le fait que les médecins surmenés courent le risque de moins bien pratiquer la médecine. ${ }^{2}$ La Déclaration de Genève intègre dorénavant ce fait en demandant aux médecins de prendre soin de leur propre santé afin de fournir les meilleurs soins possibles aux patients: «I WILL ATTEND TO my own health, well-being, and abilities in order to provide care of the highest standard.»

L'Association médicale mondiale a par ailleurs modifié l'ordre des différents paragraphes. Les obligations envers le patient sont mentionnées immédiatement après la phrase introductive, puis ce sont celles envers la profession et enfin celles envers la société.

\section{Médecine et économie}

2 https://www.wma.net/ policies-post/wmastatement-on-physcianswell-being/

Literatur zur Evidenz siehe bei Wiesing U. Ethical Aspects of Limiting Residents' Work Hours. Bioethics. 2007;21:398405.

3 Rheinsberg Z, Kloiber O, Parsa-Parsi R, Wiesing U. Medical oath: Use and relevance of the Declaration of Geneva. A survey of member organizations of the World Medical Association (WMA). Medicine, Health Care and Philosophy. 2017, doi 10.1007/s11019-017-9794-x
L'Association médicale mondiale reconnaît que la médecine doit prendre en considération la pensée économique dans de nombreux contextes. Cette pensée peut cependant se trouver dans un certain rapport de tension avec la médecine. Et ce de différentes façons: les restrictions économiques dues au manque de ressources sont, à l'échelle mondiale, les plus susceptibles de limiter la médecine, tandis que dans les pays riches, on peut observer une offre excessive et une surthérapie en raison des incitations économiques. Un serment ayant pour vocation une validité mondiale devait s'efforcer de répondre à ces différents phénomènes par une formulation aussi concise que possible. L'Association médicale mondiale a, dans la révision, mis en évidence les normes scientifiques auxquelles un médecin doit adhérer, en ajoutant à «I WILL PRACTISE my profession with conscience and dignity» la phrase «and in accordance with good medical practice». Cette mention devrait également contrecarrer toute économisation inappropriée. Les interventions inutiles, tout comme un sous-approvisionnement, ne correspondent tout simplement pas à la bonne pratique médicale.

\section{La Déclaration de Genève et sa diffusion}

La diffusion et l'utilisation du serment ne satisfont pas actuellement à l'objectif qu'il s'est lui-même fixé, à savoir être le serment de tous les médecins. Une analyse dans le contexte de la révision a constaté que la Déclaration de Genève restait à cet égard très en deçà des attentes. ${ }^{3}$ C'est notamment la raison pour laquelle l'expression "At the time of being admitted as a member of the medical profession" a été raccourcie en supprimant "At the time of being admitted". L'utilisation du serment ne se limite ainsi pas à la seule admission à la profession médicale. Le sous-titre «The Physician's Pledge» a en outre été ajouté afin de mettre en évidence cette revendication de façon encore plus claire et d'accroître la visibilité dans les nouveaux médias. La Déclaration de Genève n'était jusqu'ici pas facile à trouver par les moteurs de recherche sur Internet.

La diffusion insuffisante du serment a également été traitée lors de l'Assemblée générale de l'Association médicale mondiale pour en arriver à la conclusion que d'autres mesures devaient être prises afin de lui conférer effectivement la position à laquelle il prétend et qu'il mérite. Elle est convaincue qu'il est essentiel pour la profession d'avoir, à l'ère de la mondialisation, une norme éthique commune dans le monde entier. Des interventions sont ainsi prévues à l'occasion de conférences internationales traitant du problème d'une base éthique commune pour la profession médicale à l'ère de la mondialisation. L'Association médicale mondiale veut à l'avenir également lire le serment à l'ouverture de ses sessions annuelles.

\section{Une profession dans un monde globalisé}

La Déclaration de Genève n'est pas une fin en soi. Elle aborde un problème important de la médecine actuelle: il faut percevoir l'effort pour une plus large diffusion comme une réponse à un développement qui n'affecte pas seulement la médecine: la mondialisation.

La question se pose dans un tel monde de savoir ce qui rassemble la profession en termes éthiques et comment contrer les tendances à la déprofessionnalisation 
du service médical. Existe-t-il au niveau global un noyau de normes morales qu'un médecin doit respecter? Il ne peut y avoir qu'une réponse globale à ces questions. Les serments nationaux ne peuvent, par nature, pas apporter de réponse à ce problème.
L'Association médicale mondiale a adopté un serment pour 114 ordres et associations $d^{\text {e }}$ médecins et a ainsi établi un noyau de normes pour la plupart des associations médicales dans le monde. Ce noyau est la base essentielle et indispensable de la profession médicale.

\section{ASSOCIATION MÉDICALE MONDIALE}

\section{DÉCLARATION DE GENÈVE}

Adoptée par la $2^{\mathrm{e}}$ Assemblée Générale de l'Association Médicale Mondiale Genève (Suisse), Septembre 1948 et amendée par la $22^{\mathrm{e}}$ Assemblée Médicale Mondiale, Sydney, Australie, Août 1968 et la $35^{\mathrm{e}}$ Assemblée Médicale Mondiale, Venise, Italie, Octobre 1983 et la $46^{\mathrm{e}}$ Assemblée générale, Stockholm, Suède, Septembre 1994 et révisée par la 170 Session du Conseil, Divonne-les-Bains, France, Mai 2005

et par la $173^{\mathrm{e}}$ Session du Conseil, Divonne-les-Bains, France, Mai 2006 et amendée par la $68^{\text {ème }}$ Assemblée générale, Chicago, Etats-Unis, Octobre 2017

\section{Le Serment du médecin}

EN QUALITÉ DE MEMBRE DE LA PROFESSION MÉDICALE

JE PRENDS L'ENGAGEMENT SOLENNEL de consacrer ma vie au service de l'humanité;

JE CONSIDÉRERAI la santé et le bien-être de mon patient comme ma priorité;

JE RESPECTERAI l'autonomie et la dignité de mon patient;

JE VEILLERAI au respect absolu de la vie humaine;

JE NE PERMETTRAI PAS que des considérations d'âge, de maladie ou d'infirmité, de croyance, d'origine ethnique, de genre, de nationalité, d'affiliation politique, de race, d'orientation sexuelle, de statut social ou tout autre facteur s'interposent entre mon devoir et mon patient;

JE RESPECTERAI les secrets qui me seront confiés, même après la mort de mon patient;

J'EXERCERAI ma profession avec conscience et dignité, dans le respect des bonnes pratiques médicales;

JE PERPÉTUERAI l'honneur et les nobles traditions de la profession médicale;

JE TÉMOIGNERAI à mes professeurs, à mes collègues et à mes étudiants le respect et la reconnaissance qui leur sont dus;

JE PARTAGERAI mes connaissances médicales au bénéfice du patient et pour les progrès des soins de santé; JE VEILLERAI à ma propre santé, à mon bien-être et au maintien de ma formation afin de prodiguer des soins irréprochables;

JE N’UTILISERAI PAS mes connaissances médicales pour enfreindre les droits humains et les libertés civiques, même sous la contrainte; 